\title{
THEORY OF GALAXY FORMATION
}




\title{
GALAXY FORMATION AND EVOLUTION: WHAT TO EXPECT FROM HIERARCHICAL CLUSTERING MODELS
}

\author{
C.S. FRENK, C.M. BAUGH AND S. COLE \\ Physics Department \\ University of Durham, UK
}

\section{Introduction}

In hierarchical clustering theories of galaxy formation, galaxies form by gas cooling and condensing into dark matter halos which, in turn, form by a hierarchy of mergers (White \& Rees 1978). The context in which this process takes place is specified by a cosmological model that determines the spectrum of primordial density fluctuations and the rate at which they grow by gravitational instability. The best known example of such a model is the cold dark matter (CDM) model (see Frenk 1991 for a review), but a number of alternatives (mostly variants of CDM), have recently become popular in response to new data on large-scale structure and the COBE detection of anisotropies in the microwave background radiation. Regardless of the specific cosmological model that one wishes to consider, there are at least six distinct physical processes that need to be included in any theory of galaxy formation:

- 1. The growth of dark matter halos by accretion and mergers.

- 2. The dynamics of cooling gas.

- 3. Star formation.

- 4. Energy feedback into prestellar gas from the products of stellar evolution.

- 5. Evolution of the stellar populations that form.

- 6. Galaxy mergers.

A number of theoretical tools have been developed over the years to investigate these processes, both individually and collectively. N-body simulations have led to significant progress in understanding process (1.), while the recently developed $\mathrm{N}$-body/hydrodynamic techniques are beginning to 
address processes (1-4) and (6). In addition, semianalytic modelling, a relatively new tool, can treat all six processes together and thus explore the effects of different assumptions on the properties of the galaxy population as a whole. In this review, we will outline some of the areas where progress has been made and highlight some as yet unresolved issues.

\section{Physical processes}

\subsection{EVOLUTION OF DARK MATTER HALOS}

The main features of the formation of dark matter halos by hierarchical clustering were already established in $\mathrm{N}$-body simulations carried out a decade ago (eg. Frenk et al. 1985, 1988; Efstathiou et al. 1988). A protohalo perturbation, initially expanding at a reduced rate, collapses, often into filamentary or sheet-like structures, which subsequently break up into roughly spherical lumps. These merge together producing a centrally concentrated and essentially smooth dark halo. This process is illustrated in Figure 1 which shows the development of a galactic halo in a flat 'low'- $\Omega$ CDM model.

One of the main early results from $\mathrm{N}$-body simulations was the realisation that the rotation curves of dark galactic halos in the the standard CDM model are approximately flat, suggesting an explanation for the inferred structure of the halos of spiral galaxies (Frenk et al. 1985, Quinn et al. 1986). These simulations, however, were limited in particle number and did not resolve the inner regions of the halos where the visible galaxy actually forms. This issue has recently been addressed in a series of high-resolution simulations by Navarro, Frenk \& White (1995). The density profiles of galactic halos in the CDM model show noticeable departures from an $r^{-2}$ law, gently sloping from $r^{-1}$ near the centre to $r^{-3}$ near the virial radius. When the gravitational effect of a disk is included, the resulting rotation curves agree well with observations of galaxies, from dwarfs to bright galaxies, provided the disks fulfill two conditions: (i) their stellar mass-to-light ratio increases roughly as $L^{1 / 2}$ and (ii) the baryon fraction increases with luminosity such that for galaxies with observed circular velocity, $V_{c} \gtrsim 200 \mathrm{kms}^{-1}$, there is only a weak of correlation between this velocity and total halo mass. It is unclear whether the observed disks of spirals satisfy these conditions.

\subsection{THE DYNAMICS OF COOLING GAS}

The main ideas here were put forward nearly twenty years ago by Rees \& Ostriker (1977), Silk (1977) and Binney (1977). When a dark matter halo collapses, any gas admixed with it will also collapse, but whereas 

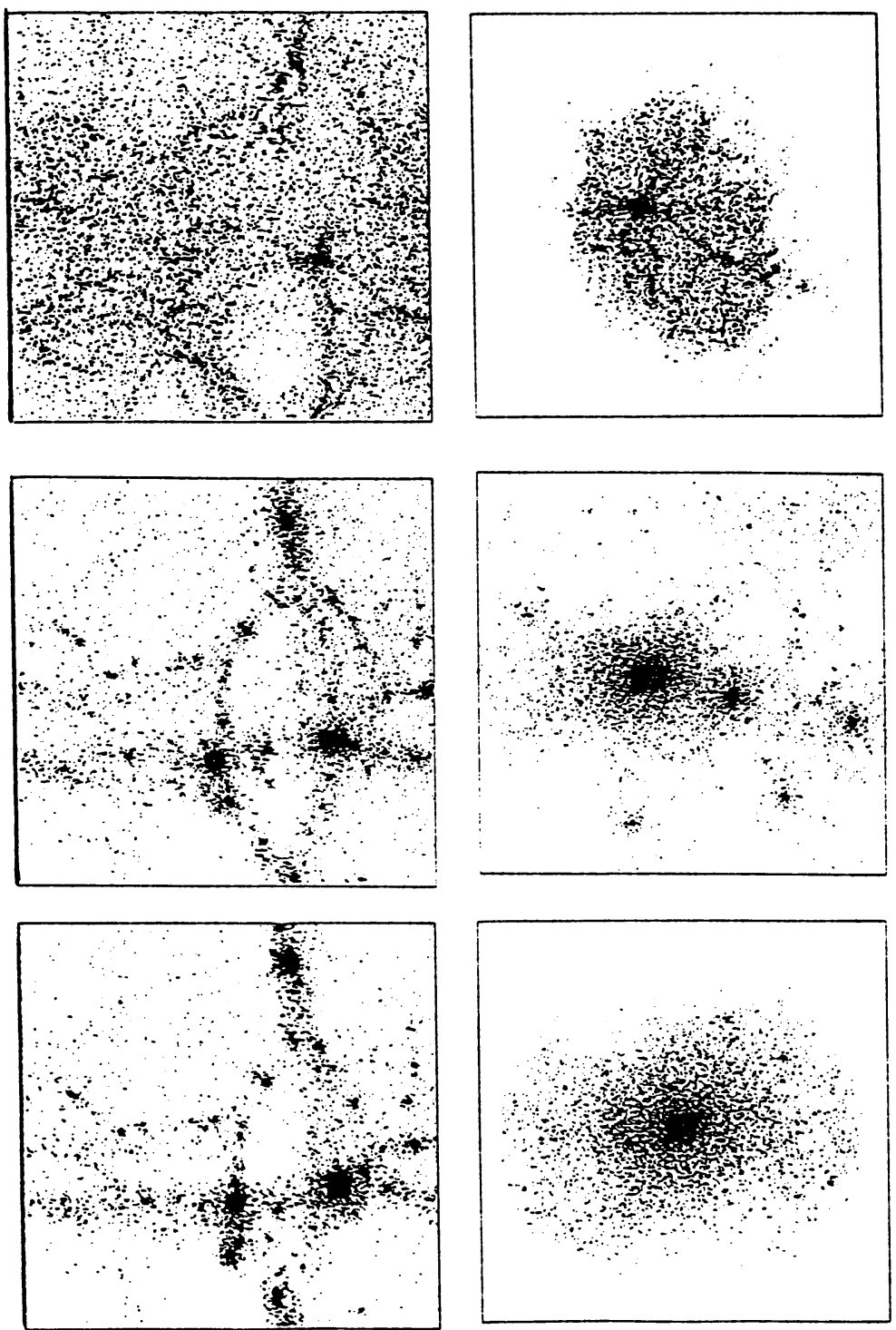

Figure 1. The formation of a galactic dark matter halo in an N-body simulation. The left-hand column shows the projected particle distribution, in comoving coordinates, of a cubical region of present comoving length $27 \mathrm{Mpc}$. The right hand column shows, now in physical coordinates, the growth of the large clump seen in the bottom left of the region. Each panel on the right hand row has length $3.8 \mathrm{Mpc}$. From top to bottom the epochs shown correspond to $z=5,0.5$ and 0 . The parameters of the simulation are: mean density, $\Omega=0.3$; cosmological constant, $\Lambda=0.7$; Hubble constant, $H_{0}=100 \mathrm{~km} \mathrm{~s}^{-1} \mathrm{Mpc}^{-1}$, with $h=0.7$; and spectrum normalisation, $\sigma_{8}=1.14$ (as inferred from the COBE data). The simulation followed 262144 particles and was performed the T3D parallel supercomputer at Edinburgh. 
the dark matter free streams, the gas is shock heated. These early papers assumed that shocks would heat up the gas to the virial temperature of the halo, an assumption verified - in the non-radiative limit - in the Nbody/hydrodynamic simulations of Evrard (1990). These and subsequent simulations (eg. Katz \& White 1993, Navarro, Frenk \& White 1995) also showed that, in this limit, the gas acquires a density profile that closely parallels that of the dark matter. Rees \& Ostriker argued that if the cooling time of virialised gas was shorter than its dynamical time, the gas would collapse to make a galaxy. White \& Rees (1978) recognized, however, that in a hierarchical model this simple scheme would lead to a cooling catastrophe since at early times the density is so high that all the gas would cool into subgalactic lumps where it would pressumably turn into stars. This patently did not happen in the universe - there is still plenty of gas around today. White \& Rees solved this problem by introducing the idea of feedback, whereby the energy released by supernovae associated with an early generation of stars reheats some of the gas before it has had a chance to condense into halos at high redshift. (Efstathiou 1992 has argued that photoionisation by a UV background at high redshift would have a similar effect.)

Testing these simple physical arguments in numerical simulations is difficult because the propensity of the gas to cool at high density implies that the behaviour of the gas is always determined by the resolution limit of the simulation. This numerical artefact, however, can be turned to advantage if it is loosely interpreted as an effective source of feedback. $\mathrm{N}$ body/hydrodynamic simulations of representative cosmological volumes in which the gas is allowed to cool are still at an early stage (eg Katz et al. 1992, Cen \& Ostriker 1992, Frenk et al. 1995) and show that the behaviour of the gas is more complex than expected in the simple analytic picture. Simulations of the formation of individual galaxies produce disks, often with beautiful spiral arms (e.g. Steinmetz \& Muller 1995), but these disks rotate much too slowly. This is because merger events transfer angular momentum from gas fragments to the outer dark matter halo in much the same way as the mergers of collionsless particles do (Navarro, Frenk \& White 1995). This angular momentum problem for disks remains a major unresolved issue in studies of galaxy formation. One possible solution may be, again, to invoke some form of feedback which might keep the gas hot and allow it to cool slowly rather to be collected in subclumps.

\subsection{STAR FORMATION AND FEEDBACK}

Current understanding of star formation and the attendant feedback, in the context of galaxy formation, is laughably poor. All that can be done 
at present is to try and model these processes in a heuristic fashion. For example, in an $\mathrm{N}$-body/hydrodynamic simulation one can stipulate a number of conditions for gas to turn into stars, eg, that it be cool and dense (ie above the Jeans mass) and that it be inflowing into a halo. Systematic tests of such algorithms are just beginning (e.g. Navarro \& White 1993).

\subsection{GALAXY MERGERS}

Simulations of the merging of individual galaxy pairs or small groups have a long and distinguished history (see Barnes's contribution elsewhere in this volume). Such simulations address issues such as the structure and rotation properties of merger remnants, or the gas flows triggered by mergers. From the point of view of galaxy formation in general, a key issue is the relative timescale for the merging of dark matter halos and the galaxies they harbour. As a consequence of their higher binding energy, galaxies take longer to merge than their halos. Furthermore, the simulations show that galaxies (or at any rate the clumps of cool gas identified with galaxies in the models) merge on a dynamical friction timescale, provided that the mass that is input into Chandrasekhar's classic formula is the total, gas plus dark matter, mass of the merging satellite (Navarro, Frenk \& White 1995).

\section{Semianalytic models}

Our understanding of the full range of complex phenomena listed in the Introduction can be approximated by a set of simple rules. These rules can then form the basis of a semianalytic model for galaxy formation, that follows the collapse and mergers of dark matter halos and the star formation histories of the galaxies. Such models (Kauffman et al. 1993, Lacey et al. 1993, Cole et al. 1994) have been successful in accounting for the general properties of the observed galaxy distribution, such as the shape of the luminosity function, faint number counts and colours. However, a number of fundamental problems remain that appear to suggest that the modelling of the processes ( 1 - 6$)$ needs to be improved, rather than altering the choice of cosmology (Heyl et al. 1994).

In the original model of Cole et al. (1994), the model galaxies were not as red as many observed ellipticals. A study of several stellar population codes (Charlot et al. 1995) has led to a revision of the Bruzual and Charlot (1993) models. This has resulted in the model galaxies being typically 0.2 mag redder in $B-K$. An updated comparison of the colour uistribution of the model galaxies from Cole et al. with observed colours is given in Figure 2.

We also present an updated versions of the redshift distributions predicted by the model of Cole et al. Figure 3 compares the model predictions 

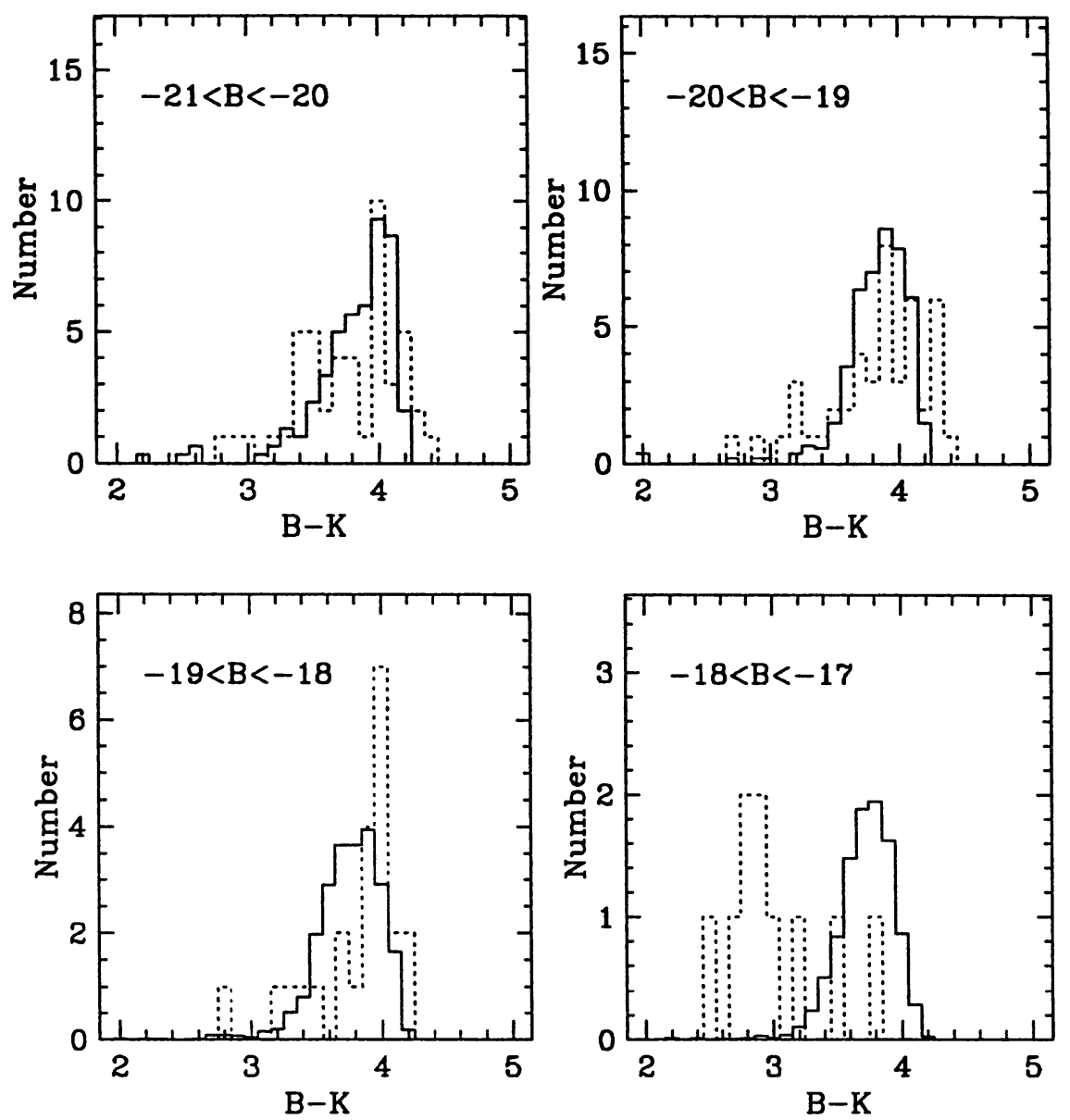

Figure 2. Histograms of B-K colour distributions for various ranges of $\mathrm{B}$ absolute magnitude. The broken lines are data from Mobasher et al. (1986) and show the observed number of galaxies in the data set. The model output is shown by the solid lines which have been normalised to enclose the same area as the data.

with the recent redshift survey data from the surveys of Glazebrook et al. $(1995 \mathrm{a}, \mathrm{b})$. The model and observed distributions are in very good agreement.

The luminosity function of Cole et al. was flatter than that achieved by other semianalytic models, because of the strong feedback adopted, which severely restricts star formation in halos of low circular velocity. However, this model still predicts more faint galaxies than are observed (Loveday et al 1992), though recent results indicate that the faint end of the luminosity function is still uncertain (McGaugh 1994, Marzke et al. 1994).

In addition, the models reproduce the observed slope and small scatter 

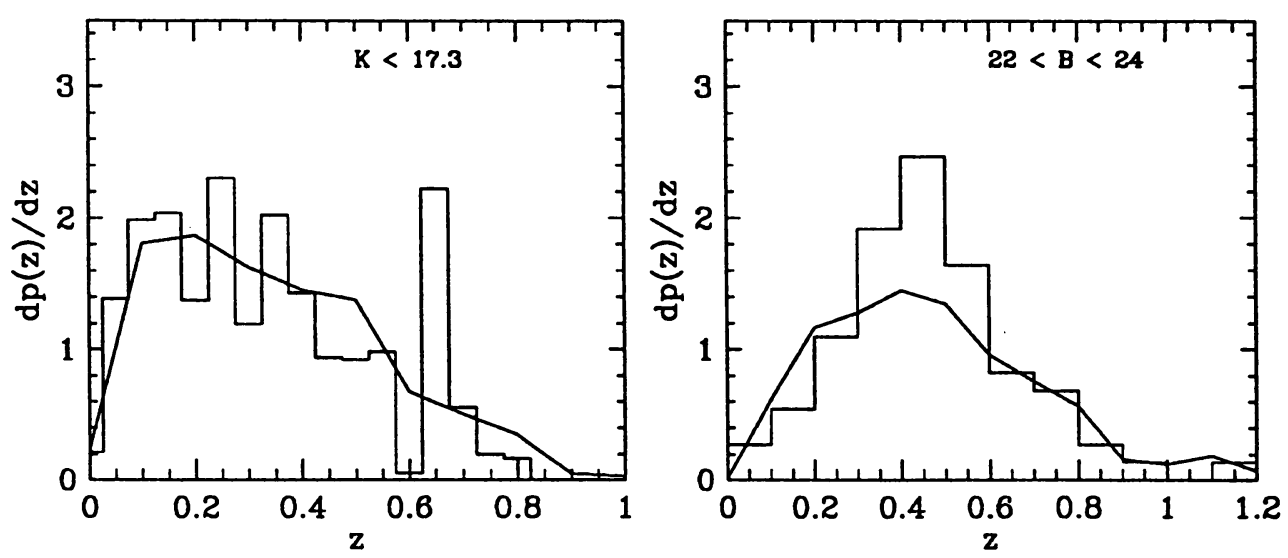

Figure 3. The redshift distribution of model galaxies compared with new data for $\mathrm{K}$ and B-band selected samples of Glazebrook et al. 1995a,b. The histograms show the observed distributions and the curves show the model galaxy redshifts. The $\mathrm{K}$ band data consists of 124 redshifts for galaxies with $K<17.3$ and is weighted for incompleteness. The B band sample is $70 \%$ complete and contains 70 galaxies with $22<B<24$.

of the observed Tully-Fisher relation between luminosity and circular velocity, but give galaxies that are either too faint or that have too high a circular velocity for their luminosity. This can be traced back to an overproduction in CDM-like cosmologies of halos typical of those that contain luminous galaxies.

Both the Tully-Fisher and luminosity function problems could be related to surface brightness effects. We plan to incorporate a scale length into the models, allowing us to select only those galaxies above some surface brightness threshold.

We have extended the model to split the light of each galaxy up into a bulge and a disk component, with the bulges formed in violent merger events. This allows us to make a broad morphological classification of our galaxies and make predictions of galaxy properties as a function of bulge to disk ratio. For example we find good agreement with the faint HST counts of Glazebrook et al. (1995c) and recover the type of evolution in cluster membership reported by Butcher \& Oemler (1978) (Baugh et al. 1995).

\section{References}

Baugh, C.M., Cole, S., Frenk, C.S., 1995, in preparation

Binney, J.J., 1977, Ap.J, 215, 483

Bruzual, G., Charlot, S., 1993, Ap.J, 405, 538

Butcher, H., Oemler, A., 1978, A.J., 219, 18

Cen, R., Ostriker, J.P., 1992, A.J., 339, 331

Charlot, S., Worthey, G., Bressan, A., 1995 A.J., in press 
Cole, S., Aragon-Salamanca, A., Frenk, C.S., Navarro, J., Zepf, S., 1994, M.N.R.A.S, 271,781

Efstathiou, G. 1992, M.N.R.A.S, 256, 43p

Efstathiou, G., Frenk, C.S., White, S.D.M., Davis, M., 1988, M.N.R.A.S, 235, 7 Evrard, A.E. 1990, Ap.J., 363, 349.

Frenk, C.S. 1991, in The Birth and Early Evolution of our Universe, Nobel Sym

No 79, eds J.S. Nilsson, et al. , World Sci., Physica Scripta, T36, 70.

Frenk, C.S., White, S.D.M., Efstathiou, G., Davis, M., 1985, Nature, 317, 595.

Frenk, C.S., White, S.D.M., Efstathiou, G., Davis, M., 1988, Ap.J., 327, 507.

Glazebrook, K., Peacock, J.A., Miler, L., Collins, C.A., 1995a, M.N.R.A.S,

275,169

Glazebrook, K., Ellis, R., Colless, M., Broadhurst, T., Allington-Smith, J.,

Tanvir, N., 1995b, M.N.R.A.S, 273, 157

Glazebrook, K., Ellis, R., Santiago, B., Griffiths, R., 1995c, M.N.R.A.S, 275, L]

Heyl, J.S., Cole, S., Frenk, C.S., Navarro, J., 1995, M.N.R.A.S, 274, 755

Katz, N., Hernquist, L., Weinberg, D.H., 1992, Ap.J, 399, L109

Katz, N., White, S.D.M., 1993, Ap.J, 412, 455

Kauffmann, G., White, S.D.M., Guiderdoni, B., 1993, M.N.R.A.S, 264, 201

Lacey, C.G., Guiderdoni, B., Rocca-Volmerange, B., Silk, J., 1993, Ap.J, 402,1:

Loveday, J., Peterson, B.A., Efstathiou, G., Maddox, S.J., 1992, Ap.J, 390, 338

Marzke, R.O., Geller, M.J., Huchra, J.P., Corwin, H.G., 1994, A.J., 108, 437

McGaugh, S., 1994, Nature, 367, 538

Mobasher, B., Sharples, R.M., Ellis, 1986, M.N.R.A.S, 223, 11

Navarro, J.F., White, S.D.M., 1993, M.N.R.A.S, 265, 271

Navarro, J.F., Frenk, C.S., White, S.D.M., 1995, M.N.R.A.S, in press

Rees, M.J., Ostriker, J.P., 1977, M.N.R.A.S, 179, 541

Silk, J., 1977 Ap.J, 211, 638

Steinmetz, M., Muller, E., 1995, M.N.R.A.S, 276, 549

Quinn, P.J., Salmon, J.K. and Zurek, W. 1986, Nature, 322, 329.

White, S.D.M., Rees, M.J. 1978, M.N.R.A.S, 183, 341.

\section{Discussion}

McGaugh: There is an extra factor of 2 in the Low Surface Brightness galaxy number density, but probably only $\sim 30 \%$ in luminosity density. What happens to the models if you turn off feedback?

Frenk: Feedback (and mergers) control the faint end slope of the luminosity function. If feedback is turned off this slope becomes unacceptably steep, with $\alpha \approx-2.5$. 